\title{
New Ostrowski-Grüss type inequalities with the derivatives bounded by functions
}

\author{
Qinghua Feng ${ }^{1,2^{*}}$ and Fanwei Meng ${ }^{2}$
}

"Correspondence: fqhua@sina.com 'School of Science, Shandong University of Technology, Zibo, Shandong 255049, China

${ }^{2}$ School of Mathematical Sciences, Qufu Normal University, Qufu,

Shandong 273165, China

\begin{abstract}
In this paper, we establish some new Ostrowski-Grüss type inequalities involving multiple interior points with the first-order derivative bounded by functions instead of constants, some of which provide sharp bounds. Then we establish a new 2D Ostrowski-Grüss type inequality involving multiple interior points with the second mixed partial derivative bounded by functions. For illustrating the applications of the Ostrowski-Grüss type inequalities established, we apply them to derive error bounds for some numerical integration formulae.
\end{abstract}

MSC: 26D10; 26D20

Keywords: Ostrowski-Grüss type inequality; sharp; numerical integration; error bound

\section{Introduction}

As is known, the Ostrowski-type inequality [1] can be used to estimate the absolute deviation of a function from its integral mean, while the Grüss inequality [2] can be used to estimate the absolute deviation of the integral of the product of two functions from the product of their respective integral. Recently, various generalizations of the Ostrowski inequality and the Grüss inequality have been established (for example, see [3-21] and the references therein). These inequalities can be used to provide explicit error bounds for numerical quadrature formulae such as the Simpson quadrature formula, trapezoid quadrature formula and so on. Among the generalizations, many Ostrowski-Grüss type inequalities have been established [13-21]. Now we list some important results in the literature.

In [13], Dragomir et al. presented an Ostrowski-Grüss type inequality for the first time as follows:

$$
\left|f(x)-\frac{1}{b-a} \int_{a}^{b} f(t) d t-\frac{f(b)-f(a)}{b-a}\left(x-\frac{a+b}{2}\right)\right| \leq \frac{1}{4}(b-a)(\Gamma-\gamma)
$$

for all $x \in[a, b]$, where $\gamma, \Gamma$ are two constants such that $\gamma \leq f^{\prime}(t) \leq \Gamma, t \in[a, b]$.

In [14], under the same conditions as above, Matic et al. improved the above result to the following form:

$$
\left|f(x)-\frac{1}{b-a} \int_{a}^{b} f(t) d t-\frac{f(b)-f(a)}{b-a}\left(x-\frac{a+b}{2}\right)\right| \leq \frac{1}{4 \sqrt{3}}(b-a)(\Gamma-\gamma) .
$$

○2013 Feng and Meng; licensee Springer. This is an Open Access article distributed under the terms of the Creative Commons Attribution License (http://creativecommons.org/licenses/by/2.0), which permits unrestricted use, distribution, and reproduction in any medium, provided the original work is properly cited. 
Then in [15], Cheng presented a sharp inequality as follows:

$$
\left|f(x)-\frac{1}{b-a} \int_{a}^{b} f(t) d t-\frac{f(b)-f(a)}{b-a}\left(x-\frac{a+b}{2}\right)\right| \leq \frac{1}{8}(b-a)(\Gamma-\gamma) .
$$

This inequality is sharp in the sense that the constant $\frac{1}{8}$ cannot be replaced by a smaller one.

In [16], Feng et al. further generalized the inequalities above, and presented a sharp Ostrowski-Grüss type inequality involving multiple interior points as follows:

$$
\begin{aligned}
& \mid \frac{1}{b-a} \sum_{i=0}^{k}\left(m_{i+1}-m_{i}\right) f\left(x_{i}\right) \\
& \quad-\frac{1}{b-a} \int_{a}^{b} f(t) d t-\frac{f(b)-f(a)}{(b-a)^{2}}\left[\frac{b^{2}-a^{2}}{2}-\sum_{i=0}^{k-1} m_{i+1}\left(x_{i+1}-x_{i}\right)\right] \mid \\
& \quad \leq \frac{1}{8}(b-a)(\Gamma-\gamma)
\end{aligned}
$$

where $x_{i} \in[a, b], i=1, \ldots, k-1$, are interior points, $m_{i} \in\left[x_{i-1}, x_{i}\right], i=1,2, \ldots, k, x_{0}=a$, $x_{k}=b, m_{0}=a, m_{k+1}=b$, and $\gamma, \Gamma$ are defined as before.

We notice that little attention is paid to the Ostrowski-Grüss type inequalities involving multiple interior points with $f^{\prime}(t)$ bounded by functions instead of constants so far in the literature. So, in this paper, motivated by the above works, we extend the Ostrowski-Grüss type inequalities to the case involving multiple interior points with the bounds of $f^{\prime}(t)$ shown as $\gamma(t) \leq f^{\prime}(t) \leq \Gamma(t), t \in[a, b]$. Some bounds will be derived based on the inequalities, and some of the bounds are sharp. A new 2D Ostrowski-Grüss type inequality will also be derived with the bounds of $\frac{\partial^{2} f(x, y)}{\partial x \partial y}$ shown as $\gamma(x, y) \leq \frac{\partial^{2} f(x, y)}{\partial x \partial y} \leq \Gamma(x, y), x \in[a, b]$, $y \in[c, d]$. We also present some applications for the Ostrowski-Grüss type inequalities established, in which new error bounds for some numerical integration formulae are derived.

\section{Main results}

Lemma 2.1 [16, Lemma 2.1] Let $I \subset R$ be an open interval, $a, b \in I, a<b . f: I \rightarrow R$ is a differential function. Furthermore, suppose that $t_{i} \in[a, b], i=0,1, \ldots, k, I_{k}: a=t_{0}<t_{1}<$ $\cdots<t_{k-1}<t_{k}=b$ is a division of the interval $[a, b]$, and $\delta_{i} \in\left[t_{i-1}, t_{i}\right], i=1,2, \ldots, k, \delta_{0}=a$, $\delta_{k+1}=b$. Then we have

$$
\sum_{i=0}^{k}\left(\delta_{i+1}-\delta_{i}\right) f\left(t_{i}\right)=\int_{a}^{b} f(t) d t+\int_{a}^{b} \zeta\left(t, I_{k}\right) f^{\prime}(t) d t,
$$

where

$$
\zeta\left(t, I_{k}\right)= \begin{cases}t-\delta_{1}, & t \in\left[t_{0}, t_{1}\right), \\ t-\delta_{2}, & t \in\left[t_{1}, t_{2}\right), \\ \cdots & \\ t-\delta_{k-1}, & t \in\left[t_{k-2}, t_{k-1}\right), \\ t-\delta_{k}, & t \in\left[t_{k-1}, t_{k}\right] .\end{cases}
$$


Lemma 2.2 Let

$$
\varphi\left(t, I_{k}\right)= \begin{cases}t-\delta_{1}-C, & t \in\left[t_{0}, t_{1}\right), \\ t-\delta_{2}-C, & t \in\left[t_{1}, t_{2}\right), \\ \cdots & \\ t-\delta_{k-1}-C, & t \in\left[t_{k-2}, t_{k-1}\right), \\ t-\delta_{k}-C, & t \in\left[t_{k-1}, t_{k}\right],\end{cases}
$$

where $C=\frac{1}{2}(b+a)-\frac{1}{b-a} \sum_{i=0}^{k-1} \delta_{i+1}\left(t_{i+1}-t_{i}\right)$. Then $\int_{a}^{b} \varphi\left(t, I_{k}\right) d t=0$.

Proof Based on the definition of $\varphi\left(t, I_{k}\right)$, we have

$$
\begin{aligned}
\int_{a}^{b} \varphi\left(t, I_{k}\right) d t & =\sum_{i=0}^{k-1}\left[\frac{\left(t_{i+1}-\delta_{i+1}-C\right)^{2}}{2}-\frac{\left(t_{i}-\delta_{i+1}-C\right)^{2}}{2}\right] \\
& =\frac{1}{2} \sum_{i=0}^{k-1}\left[\left(t_{i+1}-t_{i}\right)\left(t_{i+1}+t_{i}-2 \delta_{i+1}-2 C\right)\right] \\
& =\frac{1}{2}\left\{\sum_{i=0}^{k-1}\left(t_{i+1}^{2}-t_{i}^{2}\right)-2 \sum_{i=0}^{k-1}\left(t_{i+1}-t_{i}\right) \delta_{i+1}-2 C \sum_{i=0}^{k-1}\left(t_{i+1}-t_{i}\right)\right\} \\
& =\frac{1}{2}\left(b^{2}-a^{2}\right)-\sum_{i=0}^{k-1}\left(t_{i+1}-t_{i}\right) \delta_{i+1}-C(b-a)=0 .
\end{aligned}
$$

Theorem 2.1 Under the conditions of Lemma 2.1, if there exist two functions $\gamma(t), \Gamma(t)$ with $\gamma(t) \leq f^{\prime}(t) \leq \Gamma(t), t \in[a, b]$, then the following inequality holds:

$$
\begin{aligned}
& \left|\sum_{i=0}^{k}\left(\delta_{i+1}-\delta_{i}\right) f\left(t_{i}\right)-\int_{a}^{b} f(t) d t-\int_{a}^{b} \zeta\left(t, I_{k}\right)\left[\frac{\gamma(t)+\Gamma(t)}{2}\right] d t\right| \\
& \quad \leq \frac{1}{2}\|\Gamma-\gamma\|_{\infty}\left\{\frac{1}{2} \sum_{i=0}^{k-1}\left[t_{i+1}^{2}+t_{i}^{2}\right]-\sum_{i=0}^{k-1} \delta_{i+1}\left(t_{i+1}+t_{i}\right)+\sum_{i=0}^{k-1} \delta_{i+1}^{2}\right\} .
\end{aligned}
$$

Proof Consider

$$
\begin{aligned}
& \int_{a}^{b} \zeta\left(t, I_{k}\right)\left[f^{\prime}(t)-\frac{\gamma(t)+\Gamma(t)}{2}\right] d t \\
&=\int_{a}^{b} \zeta\left(t, I_{k}\right) f^{\prime}(t) d t-\int_{a}^{b} \zeta\left(t, I_{k}\right)\left[\frac{\gamma(t)+\Gamma(t)}{2}\right] d t \\
& \int_{a}^{b}\left|\zeta\left(t, I_{k}\right)\right| d t=\sum_{i=0}^{k-1} \int_{t_{i}}^{t_{i+1}}\left|t-\delta_{i+1}\right| d t \\
&=\sum_{i=0}^{k-1}\left[\frac{\left(t_{i+1}-\delta_{i+1}\right)^{2}}{2}+\frac{\left(t_{i}-\delta_{i+1}\right)^{2}}{2}\right] \\
&=\frac{1}{2} \sum_{i=0}^{k-1}\left[t_{i+1}^{2}+t_{i}^{2}\right]-\sum_{i=0}^{k-1} \delta_{i+1}\left(t_{i+1}+t_{i}\right)+\sum_{i=0}^{k-1} \delta_{i+1}^{2}
\end{aligned}
$$


and

$$
\begin{aligned}
\left|\int_{a}^{b} \zeta\left(t, I_{k}\right)\left[f^{\prime}(t)-\frac{\gamma(t)+\Gamma(t)}{2}\right] d t\right| & \leq \int_{a}^{b}\left|\zeta\left(t, I_{k}\right)\right|\left|\left[f^{\prime}(t)-\frac{\gamma(t)+\Gamma(t)}{2}\right]\right| d t \\
& \leq \frac{1}{2}\|\Gamma-\gamma\|_{\infty} \int_{a}^{b}\left|\zeta\left(t, I_{k}\right)\right| d t .
\end{aligned}
$$

Combining (8)-(10) and Lemma 2.1, we obtain the desired result.

Corollary 2.1 Under the conditions of Lemma 2.1, if $W_{1} \leq f(t) \leq W_{2}, t \in[a, b]$, where $W_{1}$, $W_{2}$ are two constants, then we have the following inequality:

$$
\begin{aligned}
& \left|\sum_{i=0}^{k}\left(\delta_{i+1}-\delta_{i}\right) f\left(t_{i}\right)-\int_{a}^{b} f(t) d t-\left(\frac{W_{2}+W_{1}}{2}\right)\left\{\frac{1}{2}\left(b^{2}-a^{2}\right)-\sum_{i=0}^{k-1} \delta_{i+1}\left(t_{i+1}-t_{i}\right)\right\}\right| \\
& \quad \leq\left(\frac{W_{2}-W_{1}}{2}\right)\left\{\frac{1}{2} \sum_{i=0}^{k-1}\left[t_{i+1}^{2}+t_{i}^{2}\right]-\sum_{i=0}^{k-1} \delta_{i+1}\left(t_{i+1}+t_{i}\right)+\sum_{i=0}^{k-1} \delta_{i+1}^{2}\right\} .
\end{aligned}
$$

Proof In fact, in (7) we have $\gamma(t)=W_{1}, \Gamma(t)=W_{2}$. On the other hand,

$$
\begin{aligned}
\int_{a}^{b} \zeta\left(t, I_{k}\right) d t & =\sum_{i=0}^{k-1} \int_{t_{i}}^{t_{i+1}}\left(t-\delta_{i+1}\right) d t=\sum_{i=0}^{k-1}\left[\frac{\left(t_{i+1}-\delta_{i+1}\right)^{2}}{2}-\frac{\left(t_{i}-\delta_{i+1}\right)^{2}}{2}\right] \\
& =\frac{1}{2} \sum_{i=0}^{k-1}\left[t_{i+1}^{2}-t_{i}^{2}\right]-\sum_{i=0}^{k-1} \delta_{i+1}\left(t_{i+1}-t_{i}\right) \\
& =\frac{1}{2}\left(b^{2}-a^{2}\right)-\sum_{i=0}^{k-1} \delta_{i+1}\left(t_{i+1}-t_{i}\right)
\end{aligned}
$$

Then, combining (7) and (12), we get the desired inequality (11).

Next we present a sharp Ostrowski-Grüss type inequality containing multiple interior points with $f^{\prime}(t)$ bounded by functions as follows.

Theorem 2.2 Under the conditions of Lemma 2.1, if there exist two functions $\gamma(t), \Gamma(t)$ with $\gamma(t) \leq f^{\prime}(t) \leq \Gamma(t), t \in[a, b]$, then we have the following inequality:

$$
\begin{aligned}
& \mid \sum_{i=0}^{k}\left(\delta_{i+1}-\delta_{i}\right) f\left(t_{i}\right)-\int_{a}^{b} f(t) d t-\frac{f(b)-f(a)}{b-a}\left[\frac{b^{2}-a^{2}}{2}-\sum_{i=0}^{k-1} \delta_{i+1}\left(t_{i+1}-t_{i}\right)\right] \\
& \quad-\int_{a}^{b} \varphi\left(t, I_{k}\right)\left[\frac{\Gamma(t)+\gamma(t)}{2}\right] d t \mid \\
& \quad \leq \frac{1}{2}\|\Gamma-\gamma\|_{\infty} \int_{a}^{b}\left|\varphi\left(t, I_{k}\right)\right| d t
\end{aligned}
$$

where $\varphi\left(t, I_{k}\right)$ is defined as in Lemma 2.2.

The inequality (13) is sharp in the sense that the constant $\frac{1}{2}$ on the right-hand side cannot be replaced by a smaller one. 
Proof We have the following observations:

$$
\begin{aligned}
\int_{a}^{b} \varphi\left(t, I_{k}\right)\left[f^{\prime}(t)-\frac{\Gamma(t)+\gamma(t)}{2}\right] d t \\
=\int_{a}^{b} \varphi\left(t, I_{k}\right) f^{\prime}(t) d t-\int_{a}^{b} \varphi\left(t, I_{k}\right)\left[\frac{\Gamma(t)+\gamma(t)}{2}\right] d t \\
=\sum_{i=0}^{k-1} \int_{t_{i}}^{t_{i+1}}\left(t-\delta_{i+1}-C\right) f^{\prime}(t) d t-\int_{a}^{b} \varphi\left(t, I_{k}\right)\left[\frac{\Gamma(t)+\gamma(t)}{2}\right] d t \\
=\sum_{i=0}^{k-1}\left[\left(t_{i+1}-\delta_{i+1}-C\right) f\left(t_{i+1}\right)-\left(t_{i}-\delta_{i+1}-C\right) f\left(t_{i}\right)\right] \\
\quad-\sum_{i=0}^{k-1} \int_{t_{i}}^{t_{i+1}} f(t) d t-\int_{a}^{b} \varphi\left(t, I_{k}\right)\left[\frac{\Gamma(t)+\gamma(t)}{2}\right] d t \\
=\sum_{i=0}^{k}\left(\delta_{i+1}-\delta_{i}\right) f\left(t_{i}\right)-\int_{a}^{b} f(t) d t-\frac{f(b)-f(a)}{b-a}\left[\frac{b^{2}-a^{2}}{2}-\sum_{i=0}^{k-1} \delta_{i+1}\left(t_{i+1}-t_{i}\right)\right] \\
\left.\quad-\int_{a}^{b} \varphi\left(t, I_{k}\right)\left[\frac{\Gamma(t)+\gamma(t)}{2}\right] d t, \quad\right]
\end{aligned}
$$

and

$$
\left|\int_{a}^{b} \varphi\left(t, I_{k}\right)\left[f^{\prime}(t)-\frac{\Gamma(t)+\gamma(t)}{2}\right] d t\right| \leq \frac{1}{2}\|\Gamma-\gamma\|_{\infty} \int_{a}^{b}\left|\varphi\left(t, I_{k}\right)\right| d t .
$$

Combining (14) and (15), we obtain the desired inequality (13).

To prove the sharpness of (13), we take $k=2, \delta_{0}=a, \delta_{1}=\frac{b+5 a}{6}, \delta_{2}=\frac{5 b+a}{6}, \delta_{3}=b, t_{1}=\frac{b+a}{2}$, and

$$
f(t)= \begin{cases}\sigma_{1} t, & a \leq t<\frac{b+5 a}{6}, \\ \sigma_{2} t+\frac{b+5 a}{6} \sigma_{1}-\frac{b+5 a}{6} \sigma_{2}, & \frac{b+5 a}{6} \leq t<\frac{b+a}{2}, \\ \sigma_{1} t+\frac{b+a}{2} \sigma_{2}+\frac{b+5 a}{6} \sigma_{1}-\frac{b+5 a}{6} \sigma_{2}-\frac{b+a}{2} \sigma_{1}, & \frac{b+a}{2} \leq t<\frac{5 b+a}{6} \\ \sigma_{2} t+\frac{5 b+a}{6} \sigma_{1}+\frac{b+a}{2} \sigma_{2}+\frac{b+5 a}{6} \sigma_{1}-\frac{b+5 a}{6} \sigma_{2} & \\ -\frac{b+a}{2} \sigma_{1}-\frac{5 b+a}{6} \sigma_{2}, & \frac{5 b+a}{6} \leq t \leq b\end{cases}
$$

where $\sigma_{1}, \sigma_{2}$ are two constants with $\sigma_{1}<\sigma_{2}$. Then one can see

$$
f^{\prime}(t)= \begin{cases}\sigma_{2}, & t \in\left[\frac{b+5 a}{6}, \frac{b+a}{2}\right) \cup\left[\frac{5 b+a}{6}, b\right], \\ \sigma_{1}, & t \in\left[a, \frac{b+5 a}{6}\right) \cup\left[\frac{b+a}{2}, \frac{5 b+a}{6}\right),\end{cases}
$$

and

$$
\varphi\left(t, I_{k}\right)= \begin{cases}t-\frac{b+5 a}{6}, & t \in\left[a, \frac{b+a}{2}\right) \\ t-\frac{5 b+a}{6}, & t \in\left[\frac{b+a}{2}, b\right]\end{cases}
$$


So, in fact, $\gamma(t) \equiv \sigma_{1}, \Gamma(t) \equiv \sigma_{2}$. According to the left-side hand of (15), we have

$$
\left|\int_{a}^{b} \varphi\left(t, I_{k}\right)\left[f^{\prime}(t)-\frac{\gamma(t)+\Gamma(t)}{2}\right] d t\right|=\left(\frac{\sigma_{2}-\sigma_{1}}{2}\right) \int_{a}^{b}\left|\varphi\left(t, I_{k}\right)\right| d t
$$

while according to the right-side hand of (15), we also have

$$
\begin{aligned}
\int_{a}^{b}\left|\varphi\left(t, I_{k}\right)\right|\left|\left[f^{\prime}(t)-\frac{\gamma(t)+\Gamma(t)}{2}\right]\right| d t \\
=-\int_{a}^{\frac{b+5 a}{6}} \varphi\left(t, I_{k}\right)\left|\frac{\sigma_{1}-\sigma_{2}}{2}\right| d t+\int_{\frac{b+5 a}{6}}^{\frac{b+a}{2}} \varphi\left(t, I_{k}\right)\left|\frac{\sigma_{2}-\sigma_{1}}{2}\right| d t \\
\quad-\int_{\frac{b+a}{2}}^{\frac{5 b+a}{6}} \varphi\left(t, I_{k}\right)\left|\frac{\sigma_{1}-\sigma_{2}}{2}\right| d t+\int_{\frac{5 b+a}{6}}^{b} \varphi\left(t, I_{k}\right)\left|\frac{\sigma_{1}-\sigma_{2}}{2}\right| d t \\
=\left(\frac{\sigma_{2}-\sigma_{1}}{2}\right) \int_{a}^{b}\left|\varphi\left(t, I_{k}\right)\right| d t .
\end{aligned}
$$

So, (15) holds in the equality form, which confirms the proof.

Corollary 2.2 Under the conditions of Lemma 2.1, if $W_{1} \leq f(t) \leq W_{2}, t \in[a, b]$, where $W_{1}$, $W_{2}$ are two constants, then we have the following inequality:

$$
\begin{aligned}
& \left|\sum_{i=0}^{k}\left(\delta_{i+1}-\delta_{i}\right) f\left(t_{i}\right)-\int_{a}^{b} f(t) d t-\frac{f(b)-f(a)}{b-a}\left[\frac{b^{2}-a^{2}}{2}-\sum_{i=0}^{k-1} \delta_{i+1}\left(t_{i+1}-t_{i}\right)\right]\right| \\
& \quad \leq\left(\frac{W_{2}-W_{1}}{2}\right) \int_{a}^{b}\left|\varphi\left(t, I_{k}\right)\right| d t .
\end{aligned}
$$

Remark 2.1 In Corollary 2.2, if we take $k=1, \delta_{0}=a, \delta_{1}=\frac{a+b}{2}, \delta_{2}=b, t_{0}=a, t_{1}=b$, then Corollary 2.2 becomes the trapezoid-type inequality, which provides error bound for the trapezoid quadrature formula in calculating $\int_{a}^{b} f(t) d t$.

$$
\left|\frac{f(b)+f(a)}{2}(b-a)-\int_{a}^{b} f(t) d t\right| \leq\left(\frac{W_{2}-W_{1}}{2}\right) \int_{a}^{b}\left|\varphi\left(t, I_{k}\right)\right| d t,
$$

where $\varphi\left(t, I_{k}\right)=t-\frac{a+b}{2}, t \in[a, b]$.

If we take $k=2, \delta_{0}=a, \delta_{1}=a, \delta_{2}=b, \delta_{3}=b, t_{0}=a, t_{1}=x, t_{2}=b$, then Corollary 2.2 becomes the following Ostroski-Grüss type inequality as denoted in (1) [15, Th. 1.5]:

$$
\begin{aligned}
\left|(b-a) f(x)-\int_{a}^{b} f(t) d t-[f(b)-f(a)]\left(x-\frac{a+b}{2}\right)\right| & \leq\left(\frac{W_{2}-W_{1}}{2}\right) \int_{a}^{b}\left|\varphi\left(t, I_{k}\right)\right| d t \\
& =\left(\frac{W_{2}-W_{1}}{8}\right)(b-a)^{2},
\end{aligned}
$$

where

$$
\varphi\left(t, I_{k}\right)= \begin{cases}t-a-\left(x-\frac{a+b}{2}\right), & t \in[a, x), \\ t-b-\left(x-\frac{a+b}{2}\right), & t \in[x, b] .\end{cases}
$$


If we take $k=2, \delta_{0}=a, \delta_{1}=\frac{5 a+b}{6}, \delta_{2}=\frac{a+5 b}{6}, \delta_{3}=b, t_{0}=a, t_{1}=\frac{a+b}{2}, t_{2}=b$, then Corollary 2.2 becomes the following Simpson-type inequality, which provides better bound than the corresponding result in [21] (the constant on the right-side hand of the inequality is $\frac{1}{12}$ therein).

$$
\begin{aligned}
\left|\frac{1}{3}\left[\frac{f(b)+f(a)}{2}+2 f\left(\frac{(b+a)}{2}\right)\right](b-a)-\int_{a}^{b} f(t) d t\right| & \leq\left(\frac{W_{2}-W_{1}}{2}\right) \int_{a}^{b}\left|\varphi\left(t, I_{k}\right)\right| d t \\
& =\frac{5\left(W_{2}-W_{1}\right)}{72}(b-a)^{2},
\end{aligned}
$$

where

$$
\varphi\left(t, I_{k}\right)= \begin{cases}t-\frac{5 a+b}{6}, & t \in\left[a, \frac{a+b}{2}\right) \\ t-\frac{a+5 b}{6}, & t \in\left[\frac{a+b}{2}, b\right]\end{cases}
$$

Remark 2.2 Under the conditions of Corollary 2.2, furthermore, assume that the conditions of [16, Th. 2.4] hold. Then, proceeding in the same manner as the proof in [16, Eqs. (13)-(14)], we obtain $\int_{a}^{b}\left|\varphi\left(t, I_{k}\right)\right| d t \leq \frac{(b-a)^{2}}{4}$. So, Corollary 2.2 provides better bound than the inequality in (2) [16, Th. 2.4].

In the following, we extend the result in Theorem 2.1 to $2 \mathrm{D}$ case, in which $\frac{\partial^{2} f(x, y)}{\partial x \partial y}$ is bounded by two functions.

Theorem 2.3 Let $f:[a, b] \times[c, d] \rightarrow R$ be an absolutely continuous function such that the second-order mixed partial derivative exists and there exist two functions $\gamma(x, y), \Gamma(x, y)$ with $\gamma(x, y) \leq \frac{\partial^{2} f(x, y)}{\partial x \partial y} \leq \Gamma(x, y), x \in[a, b], y \in[c, d]$. Suppose that $x_{i} \in[a, b], y_{i} \in[c, d], i=$ $0,1, \ldots, k . I_{k}: a=x_{0}<x_{1}<\cdots<x_{k-1}<x_{k}=b$ is a division of the interval $[a, b]$, while $J_{k}$ : $a=y_{0}<y_{1}<\cdots<y_{k-1}<y_{k}=d$ is a division of the interval $[c, d] . \alpha_{i} \in\left[x_{i-1}, x_{i}\right], \beta_{i} \in\left[y_{i-1}, y_{i}\right]$, $i=1,2, \ldots, k, \alpha_{0}=a, \alpha_{k+1}=b, \beta_{0}=c, \beta_{k+1}=d$. Then

$$
\begin{aligned}
& \mid\left\{\sum_{i=1}^{k-1} \sum_{j=1}^{k-1}\left(\alpha_{i+1}-\alpha_{i}\right)\left(\beta_{j+1}-\beta_{j}\right) f\left(x_{i}, y_{j}\right)+\sum_{i=1}^{k-1}\left(\alpha_{i+1}-\alpha_{i}\right)\left(y_{k}-\beta_{k}\right) f\left(x_{i}, y_{k}\right)\right. \\
& \quad-\sum_{i=1}^{k-1}\left(\alpha_{i+1}-\alpha_{i}\right)\left(y_{0}-\beta_{1}\right) f\left(x_{i}, y_{0}\right)+\sum_{j=1}^{k-1}\left(x_{k}-\alpha_{k}\right)\left(\beta_{j+1}-\beta_{j}\right) f\left(x_{k}, y_{j}\right) \\
& \quad+\left(x_{k}-\alpha_{k}\right)\left(y_{k}-\beta_{k}\right) f\left(x_{k}, y_{k}\right)-\left(x_{k}-\alpha_{k}\right)\left(y_{0}-\beta_{1}\right) f\left(x_{k}, y_{0}\right) \\
& \quad-\sum_{j=1}^{k-1}\left(x_{0}-\alpha_{1}\right)\left(\beta_{j+1}-\beta_{j}\right) f\left(x_{0}, y_{j}\right)-\left(x_{0}-\alpha_{1}\right)\left(y_{k}-\beta_{k}\right) f\left(x_{0}, y_{k}\right) \\
& \quad+\left(x_{0}-\alpha_{1}\right)\left(y_{0}-\beta_{1}\right) f\left(x_{0}, y_{0}\right)-\sum_{j=1}^{k-1} \int_{a}^{b}\left(\beta_{j+1}-\beta_{j}\right) f\left(x, y_{j}\right) d x \\
& \quad-\int_{a}^{b}\left[\left(y_{k}-\beta_{k}\right) f\left(x, y_{k}\right)-\left(y_{0}-\beta_{1}\right) f\left(x, y_{0}\right)\right] d x-\sum_{i=1}^{k-1} \int_{c}^{d}\left(\alpha_{i+1}-\alpha_{i}\right) f\left(x_{i}, y\right) d y \\
& \left.\quad-\int_{c}^{d}\left[\left(x_{k}-\alpha_{k}\right) f\left(x_{k}, y\right)-\left(x_{0}-\alpha_{1}\right) f\left(x_{0}, y\right)\right] d y+\int_{a}^{b} \int_{c}^{d} f(x, y) d y d x\right\}
\end{aligned}
$$




$$
\begin{aligned}
& -\frac{[f(b, d)-f(b, c)-f(a, d)+f(a, c)]}{(b-a)(d-c)} \\
& \times\left[\frac{1}{2}\left(b^{2}-a^{2}\right)-\sum_{i=0}^{k-1} \alpha_{i+1}\left(x_{i+1}-x_{i}\right)\right]\left[\frac{1}{2}\left(d^{2}-c^{2}\right)-\sum_{j=0}^{k-1} \beta_{j+1}\left(y_{j+1}-y_{j}\right)\right] \\
& -\int_{a}^{b} \int_{c}^{d} \phi\left(x, y, I_{k}, J_{k}\right)\left[\frac{\gamma(x, y)+\Gamma(x, y)}{2}\right] d y d x \mid \\
& \leq \frac{1}{2}\|\Gamma-\gamma\|_{\infty}\left\{\frac{1}{2} \sum_{i=0}^{k-1}\left[x_{i+1}^{2}+x_{i}^{2}\right]-\sum_{i=0}^{k-1} \alpha_{i+1}\left(x_{i+1}+x_{i}\right)+\sum_{i=0}^{k-1} \alpha_{i+1}^{2}\right\} \\
& \quad \times\left\{\frac{1}{2} \sum_{j=0}^{k-1}\left[y_{j+1}^{2}+y_{j}^{2}\right]-\sum_{j=0}^{k-1} \beta_{j+1}\left(y_{j+1}+y_{j}\right)+\sum_{j=0}^{k-1} \beta_{j+1}^{2}\right\}
\end{aligned}
$$

where

$$
\phi\left(x, y, I_{k}, J_{k}\right)=\left(x-\alpha_{i+1}\right)\left(y-\beta_{j+1}\right), \quad(x, y) \in\left[x_{i}, x_{i+1}\right) \times\left[y_{j}, y_{j+1}\right), i, j=0,1, \ldots, k-1 .
$$

Proof Similar to [16, Eq. (21)], we obtain that

$$
\begin{aligned}
\int_{a}^{b} \int_{c}^{d} \phi\left(x, y, I_{k}, J_{k}\right) \frac{\partial^{2} f(x, y)}{\partial x \partial y} d y d x \\
=\sum_{i=1}^{k-1} \sum_{j=1}^{k-1}\left(\alpha_{i+1}-\alpha_{i}\right)\left(\beta_{j+1}-\beta_{j}\right) f\left(x_{i}, y_{j}\right)+\sum_{i=1}^{k-1}\left(\alpha_{i+1}-\alpha_{i}\right)\left(y_{k}-\beta_{k}\right) f\left(x_{i}, y_{k}\right) \\
\quad-\sum_{i=1}^{k-1}\left(\alpha_{i+1}-\alpha_{i}\right)\left(y_{0}-\beta_{1}\right) f\left(x_{i}, y_{0}\right)+\sum_{j=1}^{k-1}\left(x_{k}-\alpha_{k}\right)\left(\beta_{j+1}-\beta_{j}\right) f\left(x_{k}, y_{j}\right) \\
\quad+\left(x_{k}-\alpha_{k}\right)\left(y_{k}-\beta_{k}\right) f\left(x_{k}, y_{k}\right)-\left(x_{k}-\alpha_{k}\right)\left(y_{0}-\beta_{1}\right) f\left(x_{k}, y_{0}\right) \\
\quad-\sum_{j=1}^{k-1}\left(x_{0}-\alpha_{1}\right)\left(\beta_{j+1}-\beta_{j}\right) f\left(x_{0}, y_{j}\right)-\left(x_{0}-\alpha_{1}\right)\left(y_{k}-\beta_{k}\right) f\left(x_{0}, y_{k}\right) \\
\quad+\left(x_{0}-\alpha_{1}\right)\left(y_{0}-\beta_{1}\right) f\left(x_{0}, y_{0}\right)-\sum_{j=1}^{k-1} \int_{a}^{b}\left(\beta_{j+1}-\beta_{j}\right) f\left(x, y_{j}\right) d x \\
\quad-\int_{a}^{b}\left[\left(y_{k}-\beta_{k}\right) f\left(x, y_{k}\right)-\left(y_{0}-\beta_{1}\right) f\left(x, y_{0}\right)\right] d x-\sum_{i=1}^{k-1} \int_{c}^{d}\left(\alpha_{i+1}-\alpha_{i}\right) f\left(x_{i}, y\right) d y \\
\quad \int_{c}^{d}\left[\left(x_{k}-\alpha_{k}\right) f\left(x_{k}, y\right)-\left(x_{0}-\alpha_{1}\right) f\left(x_{0}, y\right)\right] d y+\int_{a}^{b} \int_{c}^{d} f(x, y) d y d x .
\end{aligned}
$$

On the other hand, we have

$$
\begin{aligned}
& \left|\int_{a}^{b} \int_{c}^{d} \phi\left(x, y, I_{k}, J_{k}\right)\left[\frac{\partial^{2} f(x, y)}{\partial x \partial y}-\frac{\gamma(x, y)+\Gamma(x, y)}{2}\right] d y d x\right| \\
& \quad \leq \frac{1}{2} \int_{a}^{b} \int_{c}^{d}\left|\phi\left(x, y, I_{k}, J_{k}\right)\right||\Gamma(x, y)-\gamma(x, y)| d y d x
\end{aligned}
$$


and

$$
\begin{aligned}
\int_{a}^{b} & \int_{c}^{d}\left|\phi\left(x, y, I_{k}, J_{k}\right)\right| d y d x \\
= & \sum_{i=0}^{k-1} \sum_{j=0}^{k-1} \int_{x_{i}}^{x_{i+1}} \int_{y_{j}}^{y_{j+1}}\left|\left(x-\alpha_{i+1}\right)\left(t-\beta_{j+1}\right)\right| d y d x \\
= & \left\{\frac{1}{2} \sum_{i=0}^{k-1}\left[x_{i+1}^{2}+x_{i}^{2}\right]-\sum_{i=0}^{k-1} \alpha_{i+1}\left(x_{i+1}+x_{i}\right)+\sum_{i=0}^{k-1} \alpha_{i+1}^{2}\right\} \\
& \times\left\{\frac{1}{2} \sum_{j=0}^{k-1}\left[y_{j+1}^{2}+y_{j}^{2}\right]-\sum_{j=0}^{k-1} \beta_{j+1}\left(y_{j+1}+y_{j}\right)+\sum_{j=0}^{k-1} \beta_{j+1}^{2}\right\} .
\end{aligned}
$$

Combining (22)-(24), we get the desired inequality (20).

Corollary 2.3 Under the conditions of Theorem 2.3, if there exist two constants $W_{1}, W_{2}$ such that $W_{1} \leq f(x, y) \leq W_{2}$, then we have the following inequality:

$$
\begin{aligned}
& \mid\left\{\sum_{i=1}^{k-1} \sum_{j=1}^{k-1}\left(\alpha_{i+1}-\alpha_{i}\right)\left(\beta_{j+1}-\beta_{j}\right) f\left(x_{i}, y_{j}\right)+\sum_{i=1}^{k-1}\left(\alpha_{i+1}-\alpha_{i}\right)\left(y_{k}-\beta_{k}\right) f\left(x_{i}, y_{k}\right)\right. \\
& -\sum_{i=1}^{k-1}\left(\alpha_{i+1}-\alpha_{i}\right)\left(y_{0}-\beta_{1}\right) f\left(x_{i}, y_{0}\right)+\sum_{j=1}^{k-1}\left(x_{k}-\alpha_{k}\right)\left(\beta_{j+1}-\beta_{j}\right) f\left(x_{k}, y_{j}\right) \\
& +\left(x_{k}-\alpha_{k}\right)\left(y_{k}-\beta_{k}\right) f\left(x_{k}, y_{k}\right)-\left(x_{k}-\alpha_{k}\right)\left(y_{0}-\beta_{1}\right) f\left(x_{k}, y_{0}\right) \\
& -\sum_{j=1}^{k-1}\left(x_{0}-\alpha_{1}\right)\left(\beta_{j+1}-\beta_{j}\right) f\left(x_{0}, y_{j}\right)-\left(x_{0}-\alpha_{1}\right)\left(y_{k}-\beta_{k}\right) f\left(x_{0}, y_{k}\right) \\
& +\left(x_{0}-\alpha_{1}\right)\left(y_{0}-\beta_{1}\right) f\left(x_{0}, y_{0}\right)-\sum_{j=1}^{k-1} \int_{a}^{b}\left(\beta_{j+1}-\beta_{j}\right) f\left(x, y_{j}\right) d x \\
& -\int_{a}^{b}\left[\left(y_{k}-\beta_{k}\right) f\left(x, y_{k}\right)-\left(y_{0}-\beta_{1}\right) f\left(x, y_{0}\right)\right] d x-\sum_{i=1}^{k-1} \int_{c}^{d}\left(\alpha_{i+1}-\alpha_{i}\right) f\left(x_{i}, y\right) d y \\
& \left.-\int_{c}^{d}\left[\left(x_{k}-\alpha_{k}\right) f\left(x_{k}, y\right)-\left(x_{0}-\alpha_{1}\right) f\left(x_{0}, y\right)\right] d y+\int_{a}^{b} \int_{c}^{d} f(x, y) d y d x\right\} \\
& -\frac{[f(b, d)-f(b, c)-f(a, d)+f(a, c)]}{(b-a)(d-c)} \\
& \times\left[\frac{1}{2}\left(b^{2}-a^{2}\right)-\sum_{i=0}^{k-1} \alpha_{i+1}\left(x_{i+1}-x_{i}\right)\right]\left[\frac{1}{2}\left(d^{2}-c^{2}\right)-\sum_{j=0}^{k-1} \beta_{j+1}\left(y_{j+1}-y_{j}\right)\right] \\
& -\left(\frac{W_{2}+W_{1}}{2}\right)\left[\frac{1}{2}\left(b^{2}-a^{2}\right)-\sum_{i=0}^{k-1} \alpha_{i+1}\left(x_{i+1}-x_{i}\right)\right] \\
& \times\left[\frac{1}{2}\left(d^{2}-c^{2}\right)-\sum_{j=0}^{k-1} \beta_{j+1}\left(y_{j+1}-y_{j}\right)\right]
\end{aligned}
$$




$$
\begin{aligned}
\leq & \left(\frac{W_{2}-W_{1}}{2}\right)\left\{\frac{1}{2} \sum_{i=0}^{k-1}\left[x_{i+1}^{2}+x_{i}^{2}\right]-\sum_{i=0}^{k-1} \alpha_{i+1}\left(x_{i+1}+x_{i}\right)+\sum_{i=0}^{k-1} \alpha_{i+1}^{2}\right\} \\
& \times\left\{\frac{1}{2} \sum_{j=0}^{k-1}\left[y_{j+1}^{2}+y_{j}^{2}\right]-\sum_{j=0}^{k-1} \beta_{j+1}\left(y_{j+1}+y_{j}\right)+\sum_{j=0}^{k-1} \beta_{j+1}^{2}\right\} .
\end{aligned}
$$

Proof In fact, in (20) we have $\gamma(x, y)=W_{1}, \Gamma(x, y)=W_{2}$. Furthermore, we have the following observation:

$$
\begin{aligned}
\int_{a}^{b} \int_{c}^{d} \phi\left(x, y, I_{k}, J_{k}\right) d y d x \\
\quad=\sum_{i=0}^{k-1} \sum_{j=0}^{k-1} \int_{x_{i}}^{x_{i+1}} \int_{y_{j}}^{y_{j+1}}\left(x-\alpha_{i+1}\right)\left(y-\beta_{j+1}\right) d y d x \\
\quad=\left[\frac{1}{2}\left(b^{2}-a^{2}\right)-\sum_{i=0}^{k-1} \alpha_{i+1}\left(x_{i+1}-x_{i}\right)\right]\left[\frac{1}{2}\left(d^{2}-c^{2}\right)-\sum_{j=0}^{k-1} \beta_{j+1}\left(y_{j+1}-y_{j}\right)\right] .
\end{aligned}
$$

Combining (20) and (26), we obtain the desired result.

Remark 2.3 In Corollary 2.3, if we take $k=2, \alpha_{0}=a, \alpha_{1}=\frac{5 a+b}{6}, \alpha_{2}=\frac{a+5 b}{6}, \alpha_{3}=b, x_{0}=a$, $x_{1}=\frac{a+b}{2}, x_{2}=b, \beta_{0}=c, \beta_{1}=\frac{5 c+d}{6}, \beta_{2}=\frac{c+5 d}{6}, \beta_{3}=d, y_{0}=c, y_{1}=\frac{c+d}{2}, y_{2}=d$, then Corollary 2.3 becomes the 2D Simpson-type inequality.

\section{Applications to numerical quadrature formulae}

In this section, we present some applications of the results established above, and derive error bounds for some numerical quadrature formulae.

Example 1 Let $G\left(f, I_{h}\right)$ be a numerical quadrature formula for $\int_{a}^{b} f(t) d t$, and denote

$$
E(t)=\left|\int_{a}^{b} f(t) d t-G\left(f, I_{h}\right)\right|
$$

Theorem 3.1 For (27), if there exist two functions $\gamma(t), \Gamma(t)$ with $\gamma(t) \leq f^{\prime}(t) \leq \Gamma(t), t \in$ $[a, b]$, and

$$
\begin{aligned}
G\left(f, I_{h}\right)= & \sum_{i=0}^{n-1}\left\{\frac{1}{3}\left[\frac{f\left(T_{i+1}\right)+f\left(T_{i}\right)}{2}+2 f\left(\frac{\left(T_{i+1}+T_{i}\right)}{2}\right)\right] h_{i}\right. \\
& \left.-\int_{T_{i}}^{T_{i+1}} \varphi\left(t, I_{i k}\right)\left[\frac{\Gamma(t)+\gamma(t)}{2}\right] d t\right\},
\end{aligned}
$$

where $I_{h}: a=T_{0}<T_{1}<\cdots<T_{n-1}<T_{n}=b$ is a division of $[a, b], h_{i}=T_{i+1}-T_{i}, i=0,1, \ldots$, $n-1$, and

$$
\varphi\left(t, I_{i, k}\right)= \begin{cases}t-\frac{5 T_{i}+T_{i+1}}{6}, & t \in\left[T_{i}, \frac{T_{i}+T_{i+1}}{2}\right), \\ t-\frac{T_{i}+5 T_{i+1}}{6}, & t \in\left[\frac{T_{i}+T_{i+1}}{2}, T_{i+1}\right]\end{cases}
$$


then we have the following estimate:

$$
|E(t)| \leq \frac{5}{72}\|\Gamma-\gamma\|_{\infty} \sum_{i=0}^{n-1} h_{i}^{2}
$$

Proof Applying Theorem 2.2 (with $[a, b]$ replaced by $\left[T_{i}, T_{i+1}\right], k=2, \delta_{0}=T_{i}, \delta_{1}=\frac{5 T_{i}+T_{i+1}}{6}$, $\delta_{2}=\frac{T_{i}+5 T_{i+1}}{6}, \delta_{3}=T_{i+1}, t_{0}=T_{i}, t_{1}=\frac{T_{i}+T_{i+1}}{2}, t_{2}=T_{i+1}$, we obtain that

$$
\begin{aligned}
\mid \frac{1}{3} & {\left[\frac{f\left(T_{i+1}\right)+f\left(T_{i}\right)}{2}+2 f\left(\frac{\left(T_{i+1}+T_{i}\right)}{2}\right)\right] h_{i} } \\
& \quad-\int_{T_{i}}^{T_{i+1}} f(t) d t-\int_{T_{i}}^{T_{i+1}} \varphi\left(t, I_{i k}\right)\left[\frac{\Gamma(t)+\gamma(t)}{2}\right] d t \mid \\
& \leq \frac{1}{2}\|\Gamma-\gamma\|_{\infty} \int_{T_{i}}^{T_{i+1}}\left|\varphi\left(t, I_{i, k}\right)\right| d t
\end{aligned}
$$

where

$$
\varphi\left(t, I_{i, k}\right)= \begin{cases}t-\frac{5 T_{i}+T_{i+1}}{6}-C_{i}, & t \in\left[T_{i}, \frac{T_{i}+T_{i+1}}{2}\right) \\ t-\frac{T_{i}+5 T_{i+1}}{6}-C_{i}, & t \in\left[\frac{T_{i}+T_{i+1}}{2}, T_{i+1}\right]\end{cases}
$$

Furthermore,

$$
C_{i}=\frac{1}{2}\left(T_{i}+T_{i+1}\right)-\frac{1}{T_{i+1}-T_{i}} \sum_{i=0}^{1} \delta_{i+1}\left(t_{i+1}-t_{i}\right)=0,
$$

and

$$
\int_{T_{i}}^{T_{i+1}}\left|\varphi\left(t, I_{i, k}\right)\right| d t=\frac{5}{36} h_{i}^{2}
$$

Combining (30), (32) and (33) and a summation with respect to $i$ from 0 to $n-1$ yield (29)

Corollary 3.1 Under the conditions of Theorem 3.1, if there exist two constants $W_{1}, W_{2}$ with $W_{1} \leq f^{\prime}(t) \leq W_{2}, t \in[a, b], h_{i} \equiv h, i=0,1, \ldots, n-1$, and $G\left(f, I_{h}\right)$ denote the Simpson complex formula, that is,

$$
G\left(f, I_{h}\right)=\sum_{i=0}^{n-1}\left\{\frac{1}{3}\left[\frac{f\left(T_{i+1}\right)+f\left(T_{i}\right)}{2}+2 f\left(\frac{\left(T_{i+1}+T_{i}\right)}{2}\right)\right] h_{i}\right\}
$$

then we have

$$
|E(t)| \leq \frac{5}{72}\left(W_{2}-W_{1}\right)(b-a) h
$$

Proof Considering $\int_{T_{i}}^{T_{i+1}} \varphi\left(t, I_{i, k}\right) d t=0$ and $b-a=n h$, from (29) we can obtain (33). 
Example 2 Let $G\left(f, I_{h}, J_{h}\right)$ be a numerical quadrature formula for $\int_{a}^{b} \int_{c}^{d} f(x, y) d x d y$, and denote

$$
E(x, y)=\left|\int_{a}^{b} \int_{c}^{d} f(x, y) d x d y-G\left(f, I_{h}, J_{h}\right)\right|
$$

Theorem 3.2 For (35), if there exist two functions $\gamma(x, y), \Gamma(x, y)$ with $\gamma(x, y) \leq \frac{\partial^{2} f(x, y)}{\partial x \partial y} \leq$ $\Gamma(x, y), x \in[a, b], y \in[c, d]$, and

$$
\begin{aligned}
G\left(f, I_{h}, J_{h}\right)= & \sum_{i=0}^{n-1} \sum_{j=0}^{n-1}\left\{-\frac{\left(x_{i+1}-x_{i}\right)\left(y_{j+1}-y_{j}\right)}{9}\left[f\left(x_{i}, \frac{y_{j}+y_{j+1}}{2}\right)+f\left(x_{i+1}, \frac{y_{j}+y_{j+1}}{2}\right)\right.\right. \\
& \left.+f\left(\frac{x_{i}+x_{i+1}}{2}, y_{j}\right)+f\left(\frac{x_{i}+x_{i+1}}{2}, y_{j+1}\right)+4 f\left(\frac{x_{i}+x_{i+1}}{2}, \frac{y_{j}+y_{j+1}}{2}\right)\right] \\
& -\frac{\left(x_{i+1}-x_{i}\right)\left(y_{j+1}-y_{j}\right)}{36}\left[f\left(x_{i}, y_{j}\right)+f\left(x_{i}, y_{j+1}\right)+f\left(x_{i+1}, y_{j}\right)+f\left(x_{i+1}, y_{j+1}\right)\right] \\
& +\frac{\left(y_{j+1}-y_{j}\right)}{6} \int_{x_{i}}^{x_{i+1}}\left[f\left(x, y_{j}\right)+f\left(x, \frac{y_{j}+y_{j+1}}{2}\right)+f\left(x, y_{j+1}\right)\right] d x \\
& +\frac{\left(x_{i+1}-x_{i}\right)}{6} \int_{y_{j}}^{y_{j+1}}\left[f\left(x_{i}, y\right)+f\left(\frac{x_{i}+x_{i+1}}{2}, y\right)+f\left(x_{i+1}, y\right)\right] d y \\
& \left.+\int_{x_{i}}^{x_{i+1}} \int_{y_{j}}^{y_{j+1}} \phi\left(s, t, I_{i, h}, J_{j, h}\right)\left[\frac{\gamma(x, y)+\Gamma(x, y)}{2}\right] d y d x\right\},
\end{aligned}
$$

where $I_{h}: a=X_{0}<X_{1}<\cdots<X_{n-1}<X_{n}=b$ is a division of the interval $[a, b]$, while $J_{h}: a=$ $Y_{0}<Y_{1}<\cdots<Y_{n-1}<Y_{n}=d$ is a division of the interval $[c, d], h_{1, i}=x_{i+1}-x_{i}, h_{2, j}=y_{j+1}-y_{j}$, $i, j=0,1, \ldots, n-1$, and

$$
\phi\left(x, y, I_{i, h}, J_{j, h}\right)=\left\{\begin{array}{c}
\left(x-\frac{5 X_{i}+X_{i+1}}{6}\right)\left(y-\frac{5 Y_{j}+Y_{j+1}}{6}\right), \\
x \in\left[X_{i}, \frac{X_{i}+X_{i+1}}{2}\right), y \in\left[Y_{j}, \frac{Y_{j+}+Y_{j+1}}{2}\right), \\
\left(x-\frac{5 X_{i}+X_{i+1}}{6}\right)\left(y-\frac{Y_{j+5} Y_{j+1}}{6}\right), \\
x \in\left[X_{i}, \frac{X_{i}+X_{i+1}}{2}\right), y \in\left[\frac{Y_{j}+Y_{j+1}}{2}, Y_{j+1}\right], \\
\left(x-\frac{X_{i}+5 X_{i+1}}{6}\right)\left(y-\frac{5 Y_{j}+Y_{j+1}}{6}\right), \\
x \in\left[\frac{X_{i}+X_{i+1}}{2}, X_{i+1}\right], y \in\left[Y_{j}, \frac{Y_{j+} Y_{j+1}}{2}\right), \\
\left(x-\frac{X_{i}+5 X_{i+1}}{6}\right)\left(y-\frac{Y_{j+5} Y_{j+1}}{6}\right), \\
x \in\left[\frac{X_{i}+X_{i+1}}{2}, X_{i+1}\right], y \in\left[\frac{Y_{j+} Y_{j+1}}{2}, Y_{j+1}\right],
\end{array}\right.
$$

then we have the following estimate:

$$
|E(x, y)| \leq \frac{25}{2592}\|\Gamma-\gamma\|_{\infty}\left(\sum_{i=0}^{n-1} h_{1, i}^{2}\right)\left(\sum_{i=0}^{n-1} h_{2, i}^{2}\right) .
$$

The desired result can be obtained by a suitable application of Theorem 2.3 (with $[a, b]$ replaced by $\left[x_{i}, x_{i+1}\right],[c, d]$ replaced by $\left[y_{j}, y_{j+1}\right], k=2, \alpha_{0}=x_{i}, \alpha_{1}=\frac{5 x_{i}+x_{i+1}}{6}, \alpha_{2}=\frac{x_{i}+5 x_{i+1}}{6}$, $\alpha_{3}=x_{i+1}, x_{0}=x_{i}, x_{1}=\frac{x_{i}+x_{i+1}}{2}, x_{2}=x_{i+1}, \beta_{0}=y_{j}, \beta_{1}=\frac{5 y_{j}+y_{j+1}}{6}, \beta_{2}=\frac{y_{j}+5 y_{j+1}}{6}, \beta_{3}=y_{j+1}, y_{0}=y_{j}$, $y_{1}=\frac{y_{j}+y_{j+1}}{2}, y_{2}=y_{j+1}$ ), and a summation with respect to $i, j$ from 0 to $n-1$. 
In Theorem 3.2, after a simple computation, one can see $\int_{x_{i}}^{x_{i+1}} \int_{y_{j}}^{y_{j+1}} \phi\left(x, y, I_{i, h}, J_{j, h}\right) d y d x=$ 0 . So, we obtain the following corollary.

Corollary 3.2 Under the conditions of Theorem 3.2, if there exist two constants $W_{1}, W_{2}$ with $W_{1} \leq \frac{\partial^{2} f(x, y)}{\partial x \partial y} \leq W_{2}, x \in[a, b], y \in[c, d], h_{1, i}=h_{2, j} \equiv h, i, j=0,1, \ldots, n-1$, and $G\left(f, I_{h}, J_{h}\right)$ denote the $2 D$ Simpson complex formula, that is,

$$
\begin{aligned}
G\left(f, I_{h}, J_{h}\right)= & \sum_{i=0}^{n-1} \sum_{j=0}^{n-1}\left\{-\frac{\left(x_{i+1}-x_{i}\right)\left(y_{j+1}-y_{j}\right)}{9}\left[f\left(x_{i}, \frac{y_{j}+y_{j+1}}{2}\right)+f\left(x_{i+1}, \frac{y_{j}+y_{j+1}}{2}\right)\right.\right. \\
& \left.+f\left(\frac{x_{i}+x_{i+1}}{2}, y_{j}\right)+f\left(\frac{x_{i}+x_{i+1}}{2}, y_{j+1}\right)+4 f\left(\frac{x_{i}+x_{i+1}}{2}, \frac{y_{j}+y_{j+1}}{2}\right)\right] \\
& -\frac{\left(x_{i+1}-x_{i}\right)\left(y_{j+1}-y_{j}\right)}{36}\left[f\left(x_{i}, y_{j}\right)+f\left(x_{i}, y_{j+1}\right)+f\left(x_{i+1}, y_{j}\right)+f\left(x_{i+1}, y_{j+1}\right)\right] \\
& +\frac{\left(y_{j+1}-y_{j}\right)}{6} \int_{x_{i}}^{x_{i+1}}\left[f\left(x, y_{j}\right)+f\left(x, \frac{y_{j}+y_{j+1}}{2}\right)+f\left(x, y_{j+1}\right)\right] d x \\
& \left.+\frac{\left(x_{i+1}-x_{i}\right)}{6} \int_{y_{j}}^{y_{j+1}}\left[f\left(x_{i}, y\right)+f\left(\frac{x_{i}+x_{i+1}}{2}, y\right)+f\left(x_{i+1}, y\right)\right] d y\right\}
\end{aligned}
$$

then we have the following estimate:

$$
|E(x, y)| \leq \frac{25}{2592}\left(W_{2}-W_{1}\right)(b-a)(d-c) h^{2} .
$$

\section{Conclusions}

We have established some new Ostrowski-Grüss type inequalities involving multiple interior points with the derivatives bounded by functions in both 1D and 2D cases and derived some sharp bounds related to them. As one can see, the inequalities are of new forms and provide better bounds than some existing results in the literature. As for applications, new error bounds for some numerical quadrature formulae are derived based on the inequalities established.

\section{Competing interests}

The authors declare that they have no competing interests.

\section{Authors' contributions}

QF carried out the main part of this article. All authors read and approved the final manuscript.

\section{Acknowledgements}

The authors would like to thank the referees very much for their valuable suggestions on improving this paper.

Received: 2 March 2013 Accepted: 3 September 2013 Published: 07 Nov 2013

\section{References}

1. Ostrowski, A: Über die Absolutabweichung einer differentiierbaren Funktion von ihren Integralmittelwert. Comment. Math. Helv. 10, 226-227 (1938)

2. Grüss, G: Über das Maximum des absoluten Betrages von $[1 /(b-a)] \int_{a}^{b} f(x) g(x) d x-\left[1 /(b-a)^{2}\right] \int_{a}^{b} f(x) d x \int_{a}^{b} g(x) d x$. Math. Z. 39, 215-226 (1935)

3. Alomari, M, Darus, M, Dragomir, SS, Cerone, P: Ostrowski type inequalities for functions whose derivatives are s-convex in the second sense. Appl. Math. Lett. 23, 1071-1076 (2010)

4. Anastassiou, GA: High order Ostrowski type inequalities. Appl. Math. Lett. 20, 616-621 (2007)

5. Tseng, KL, Hwang, SR, Yang, GS, Chou, YM: Improvements of the Ostrowski integral inequality for mappings of bounded variation I. Appl. Math. Comput. 217, 2348-2355 (2010)

6. Dragomir, SS: The discrete version of Ostrowski inequalities in normed linear spaces. J. Inequal. Pure Appl. Math. 3(1), Article ID 2 (2002) 
7. Dragomir, SS, Sofo, A: An inequality for monotonic functions generalizing Ostrowski and related results. Comput. Math. Appl. 51, 497-506 (2006)

8. Cerone, P, Cheung, WS, Dragomir, SS: On Ostrowski type inequalities for Stieltjes integrals with absolutely continuous integrands and integrators of bounded variation. Comput. Math. Appl. 54, 183-191 (2007)

9. Liu, Z: Some Ostrowski type inequalities. Math. Comput. Model. 48, 949-960 (2008)

10. Pachpatte, BG: On an inequality of Ostrowski type in three independent variables. J. Math. Anal. Appl. 249, 583-591 (2000)

11. Xue, QL, Zhu, J, Liu, WJ: A new generalization of Ostrowski-type inequality involving functions of two independent variables. Comput. Math. Appl. 60, 2219-2224 (2010)

12. Aljinović, AA, Pečarić, J: Discrete weighted Montgomery identity and discrete Ostrowski type inequalities. Comput. Math. Appl. 48, 731-745 (2004)

13. Dragomir, SS, Wang, S: An inequality of Ostrowski-Grüss' type and its applications to the estimation of error bounds for some special means and for some numerical quadrature rules. Comput. Math. Appl. 33(11), 15-20 (1997)

14. Ujević, N: New bounds for the first inequality of Ostrowski-Grüss type and applications. Comput. Math. Appl. 46, 421-427 (2003)

15. Cheng, XL: Improvement of some Ostrowski-Grüss type inequalities. Comput. Math. Appl. 42, 109-114 (2001)

16. Feng, QH, Meng, FW: Some generalized Ostrowski-Grüss type integral inequalities. Comput. Math. Appl. 63, 652-659 (2012)

17. Niezgoda, M: A new inequality of Ostrowski-Grüss type and applications to some numerical quadrature rules. Comput. Math. Appl. 58, 589-596 (2009)

18. Matić, M, Pečarić, J, Ujević, N: Improvement and further generalization of inequalities of Ostrowski-Grüss type Comput. Math. Appl. 39, 161-175 (2000)

19. Liu, Z: Some Ostrowski-Grüss type inequalities and applications. Comput. Math. Appl. 53, $73-79$ (2007)

20. Yang, S: A unified approach to some inequalities of Ostrowski-Grüss type. Comput. Math. Appl. 51, 1047-1056 (2006)

21. Pearce, CEM, Pečarić, J, Ujević, N, Varošanec, S: Generalizations of some inequalities of Ostrowski-Grüss type. Math. Inequal. Appl. 3(1), 25-34 (2000)

10.1186/1029-242X-2013-456

Cite this article as: Feng and Meng: New Ostrowski-Grüss type inequalities with the derivatives bounded by

functions. Journal of Inequalities and Applications 2013, 2013:456

\section{Submit your manuscript to a SpringerOpen ${ }^{\circ}$ journal and benefit from:}

- Convenient online submission

Rigorous peer review

- Immediate publication on acceptance

- Open access: articles freely available online

- High visibility within the field

- Retaining the copyright to your article 